\title{
DESIGN ISSUES FOR CRYOGENIC COOLING OF SHORT PERIOD SUPERCONDUCTING UNDULATORS
}

\author{
M. A. Green, D. R. Dietderich, S. Marks, S. O. Prestemon, \\ and R. D. Schlueter \\ Lawrence Berkeley National Laboratory, Berkeley CA 94720, USA
}

\begin{abstract}
Superconducting insertion devices, which produce periodic magnetic fields, have been built and installed in a number of synchrotron-light source storage-rings. For the most part, these devices have been wigglers, which have relatively long period lengths. This report concerns itself with the special cryogenic issues associated with short period undulators. The motivation for considering the incorporation of superconducting technology in insertion device designs is to achieve higher magnetic fields than can be achieved with more conventional permanent magnet technology. Since the peak field decreases sharply with increased magnet gap to period ratio, the cryogenic design of the magnet system is crucial. In particular, the insulation required for a warm vacuum bore device is impractical for short period undulators. This report describes the issues that are related to a cold bore $(\sim 4 \mathrm{~K})$ and an intermediate temperature bore $(30$ to $70 \mathrm{~K})$ designs. The criteria for the use of small cryocoolers for cooling a short period undulator are presented. The problems associated with connecting small coolers to an undulator at $4.2 \mathrm{~K}$ are discussed.
\end{abstract}

\section{INTRODUCTION TO COOLING SUPERCONDUCTING UNDULATORS}

This report discusses options for cooling superconducting insertion devices. At least three types of insertion devices are found on modern light sources. They are bending magnets, wigglers, and undulators. Bending magnets can be considered as insertion devices that are an integral part of the machine lattice. The Superbend dipoles on the ALS at the Lawrence Berkeley Laboratory are an example of the first type of a bending magnet insertion device [1]. Wigglers and undulators, on the other hand, often do not affect the beam dynamics of the machine in a large way. These devices can often be shut off without affecting the performance of the light source.

Most light source rings do not have large numbers of devices that operate at cryogenic temperatures below $5 \mathrm{~K}$. The choice of the type of cryogenic refrigeration system is often dictated by the number of low temperature devices in the ring and the size of the total heat load. When there are a few devices that operate at $4 \mathrm{~K}$, the least expensive cooling system is often individual $4 \mathrm{~K}$ coolers on each device [1]. For increased reliability, the cryogenic coolers may be backed up with liquid helium and liquid nitrogen.

A wiggler or an undulator is a bending magnet with bends of alternating polarity. The net bending of the electron beam as it goes through a wiggler or an undualtor is zero. As a result, these types of devices have only a small effect on the beam dynamics of the storage ring as whole, which is considered to be desirable. 
The differences between a wiggler and an undulator are subtle. In general an alternating field insertion device is a wiggler when the bend in each half-cell (a section where the field has the same polarity) is much greater than the synchrotron light generation angle $\gamma^{-1}$. The symbol $\gamma$ is defined as the ratio of the accelerated electron mass to the rest mass of an electron. (For the ALS at an electron energy of $1.9 \mathrm{GeV}, \gamma=3718$ ). An alternating field insertion device is an undulator when the bend per half-cell is much less than the synchrotron-light generation angle $\gamma^{-1}$. A wiggler or an undulator can be defined in terms of a dimensionless constant $\mathrm{K}$ that represents the ratio of the electron-bending angle to the light generation angle. A definition for $\mathrm{K}$ given in SI units is given as follows [2],[3] [4];

$$
K=\frac{e B_{o} \lambda_{o}}{2 \pi m_{e} c}=93.4 B_{o} \lambda_{o}
$$

where $\mathrm{e}$ is the charge of an electron $\left(1.6022 \times 10^{-19} \mathrm{C}\right) ; \mathrm{B}_{\mathrm{o}}$ is the peak value of the sinusoidal magnetic induction in an insertion device cell; and $\lambda_{u}$ is the cell length (the insertion device period from the peak field point of the field at one polarity to the next peak field point of the same polarity). The value of $\mathrm{m}_{\mathrm{e}}$ is the mass of an electron $\left(\mathrm{m}_{\mathrm{e}}=9.1095 \times 10^{-31} \mathrm{~kg}\right)$; and c is the velocity of light $\left(\mathrm{c}=2.998 \times 10^{8} \mathrm{~m} \mathrm{~s}^{-1}\right)$. The peak magnetic induction $\mathrm{B}_{\mathrm{o}}$ is given in $\mathrm{T}$; the insertion device period $\lambda_{u}$ is given in meters. When $K \gg 1$ the device is definitely a wiggler because the light from the device is incoherent. When $\mathrm{K} \leq 1$ the device produces a coherent light beam like a laser. This device is an undulator. When $\mathrm{K}$ is near 1 , there is enough coherence in the light to make the beam interesting to material scientists. Most undulators that are of interest to the light source community have a $\mathrm{K}$ around 1.

For a device to be an undulator the period $\lambda$ of the magnetic field must be short and / or the magnetic field must be low. High field undulators produce shorter wave-lengths of light, so the light source community wants to have undulators with a short period (say $\lambda_{\mathrm{o}}<15 \mathrm{~mm}$ ) and a high on-axis induction $\left(\mathrm{B}_{\mathrm{o}}>1 \mathrm{~T}\right)$. In order to achieve this kind of performance in an undulator, the magnet gap $\mathrm{G}_{\mathrm{M}}$ must be small (say $\mathrm{G}_{\mathrm{M}}<10 \mathrm{~mm}$ ) and the current density in the undulator superconducting coils must be high (say $\mathrm{j}>1500 \mathrm{~A} \mathrm{~mm}^{-2}$ ) [5].

\section{THE UNDULATOR BORE TEMPERATURE IS A DESIGN FACTOR}

The only way to reduce the magnet gap $G_{M}$ for a given vacuum chamber gap $G$ is to reduce the thickness of the beam tube and the insulation between the beam tube and the iron pole of the magnet. FIG 1 shows a typical beam tube for an insertion device magnet.

The thickness of the beam tube $\mathrm{h}$ is proportional to the width of the beam tube $\mathrm{W}$ and it is inversely proportional to the square root of the design stress for the material in the beam tube. The thickness of the beam tube $h$ will typically be 0.015 to 0.03 times the beam tube width $\mathrm{W}$, depending on the beam tube material. For a design beam gap $\mathrm{G}=5 \mathrm{~mm}$, an aluminum beam tube thickness $\mathrm{h}$ would be about $0.9 \mathrm{~mm}$.

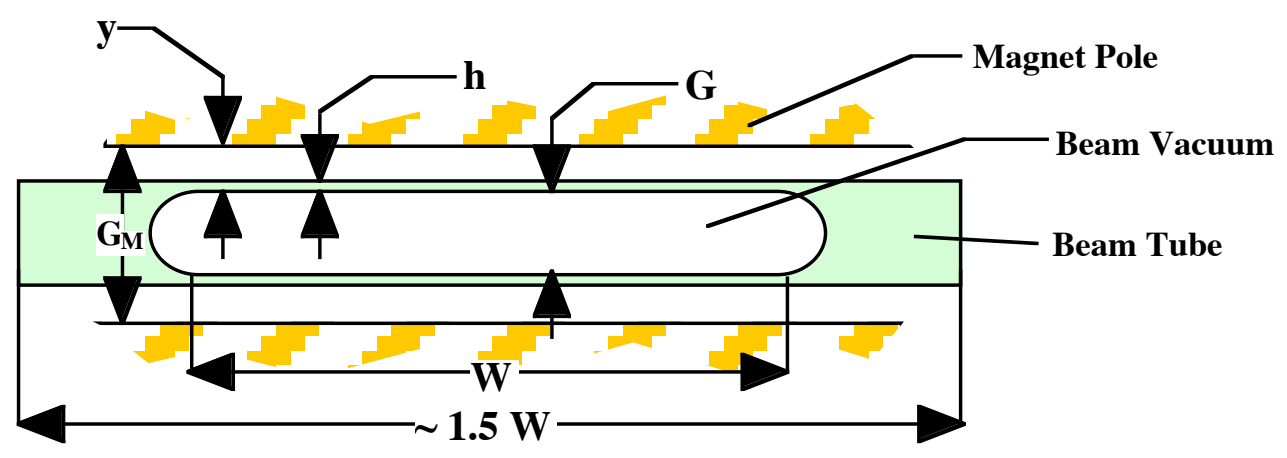

FIGURE 1. A cross section of typical undulator magnet beam tube showing the tube thickness as a function of the beam region width $\mathrm{W}$. For a typical undulator magnet beam tube $\mathrm{W}=8 \mathrm{G}$. 
TABLE 1. The insertion device magnet gap $\mathrm{G}_{\mathrm{M}}$ between the poles as a function of the beam vacuum chamber gap $\mathrm{G}$ and the temperature of the beam vacuum tube $\mathrm{T}$ (See reference [6])

\begin{tabular}{ccccc} 
Beam Tube T & \multicolumn{4}{c}{ The Magnet Gap between the Insertion Device Poles $\mathbf{G}_{\mathbf{M}}(\mathrm{mm})$} \\
& $\mathrm{G}=5 \mathrm{~mm}$ & $\mathrm{G}=10 \mathrm{~mm}$ & $\mathrm{G}=15 \mathrm{~mm}$ & $\mathrm{G}=20 \mathrm{~mm}$ \\
$300 \mathrm{~K}$ & 15.8 & 22.6 & 29.2 & 36.2 \\
30 to $80 \mathrm{~K}$ & 10.2 & 17.0 & 23.8 & 30.6 \\
$4.4 \mathrm{~K}$ & 7.6 & 15.2 & 22.8 & 30.4
\end{tabular}

The following is assumed in the table above. The beam tube width $\mathrm{W}=8 \mathrm{G}$. The beam vacuum tube is made from 6061 aluminum with a design stress of $104 \mathrm{MPa}$. Ten layers of MLI are used for the $300 \mathrm{~K}$ bore case; three layers of MLI are used for the 30 to $80 \mathrm{~K}$ bore case. There is no MLI at $4.4 \mathrm{~K}$. Each MLI layer is $0.4 \mathrm{~mm}$ thick. An additional space of $0.5 \mathrm{~mm}$ is added for vacuum pumping. At $4.4 \mathrm{~K}$, the beam tube forms part of the cryostat helium vessel with a design pressure of $0.25 \mathrm{MPa}$.

TABLE 1 shows the calculated value of the insertion magnet gap $\mathrm{G}_{\mathrm{M}}$ as a function of the beam gap $\mathrm{G}$ for beam tube temperatures of $300 \mathrm{~K}, 30$ to $80 \mathrm{~K}$, and $4.4 \mathrm{~K}$. It is clear that the effect of beam tube temperature on the insertion device magnet performance is greatest for the smallest beam gaps, because the magnetic field generated by the device on the beam axis is approximately inversely proportional to the magnet physical gap $\mathrm{G}_{\mathrm{M}}$. For devices with a larger beam gap (say greater than $10 \mathrm{~mm}$ ) there is not a strong reason for pushing the beam tube temperature clear down to $4.4 \mathrm{~K}$. This is particularly true when one considers the effect of beam heating on the selection of the type of refrigeration for the insertion device. For small beam gaps (say $<5 \mathrm{~mm}$ ), cooling the beam tube to $4.4 \mathrm{~K}$ appears to be advantageous.

\section{BEAM HEATING OF A COLD BEAM VACUUM CHAMBER}

Heating of a cold beam vacuum chamber can come from a number of sources such as thermal conduction into the chamber from the ends, radiation heat transfer from the ends, beam scraping, synchrotron radiation heating, and heating due to beam image currents in the beam tube. Heat into the cold beam tube from most of the sources can be controlled so that the level of heating is only a few watts [6]. Heating due to image currents from the beam may be the largest source of heat into a cold insertion device bore tube.

Beam heating is due to currents induced on the inner surface of the bore tube due to the passage of the electron beam bunches. The induced currents are within a skin depth of the inner surface of the bore tube. There are two skin depths to be considered the ordinary skin depth and the anomalous skin depth [7]. The ordinary skin depth varies as the square root of the bore tube material resistivity $\rho$ and the frequency of the beam $\omega$. The anomalous skin depth kicks in when the ordinary skin depth is less than the mean free path for the electrons flowing in the metal lining of the bore tube [8]. The anomalous skin depth does not change with $\rho$, so the heating calculated using the anomalous skin depth represents the lower limit for the beam heating in the beam tube. For the range of RF frequencies of interest for beams in light sources, the ordinary skin depth applies at beam tube temperatures above $70 \mathrm{~K}$. For low temperatures $(<50 \mathrm{~K})$, the anomalous skin depth effect may apply depending on the residual resistance ratio (RRR) of the material that lines the beam tube inner surface, assuming that this material is at least one skin depth thick. An example of material that might line a beam tube is a layer of pure copper plated on the inside of a stainless steel bore tube. This layer of cooper reduces the beam heating because it has a much lower resistivity than the stainless steel that makes up the rest of the beam tube.

The beam heating per unit length of a light source beam tube that is at a high enough temperature for the ordinary skin depth equation to apply can be calculated using the following expression [7];

$$
\frac{P}{\ell}=\Gamma\left(\frac{3}{4}\right) \frac{2^{1 / 2}}{8 \pi^{2}} \frac{C_{M} \mu^{1 / 2} \rho^{1 / 2} c^{1 / 2}}{R L_{B}^{3 / 2}} \frac{I^{2}}{m}
$$


where $C_{M}$ is the machine circumference; $\mu$ is the permeability of the beam tube material; $\rho$ is the electrical resistivity of the bore tube material, $\mathrm{c}$ is the velocity of light, $\mathrm{I}$ is the beam current in the storage ring; $R$ is the radius of the beam tube $(R=G / 2) ; L_{B}$ is the beam bunch length and $\mathrm{m}$ is the number of beam bunches in the ring. $\Gamma(3 / 4)$ is the gamma function of $3 / 4$. $(\Gamma(3 / 4)=1.2254)$

The minimum beam heat per unit length where the anomalous skin depth applies (at low temperatures and high values of RRR for the beam tube material) can be calculated using the following expression [7];

$$
\frac{P}{\ell}=\Gamma\left(\frac{5}{6}\right) \frac{3^{1 / 6}}{8 \pi^{2}(2 \pi)^{1 / 3}} \frac{C_{M} c^{2 / 3} \mu^{2 / 3}(\rho \lambda)^{1 / 3}}{R L_{B}^{5 / 3}} \frac{I^{2}}{m}
$$

where the quantity $\rho \lambda$ is a constant of the bore material that is independent of temperature. (For copper $\rho \lambda=6.52 \times 10^{-16} \Omega \mathrm{m}^{2}$, and for aluminum $\rho \lambda=3.97 \times 10^{-16} \Omega \mathrm{m}^{2}$.) $\Gamma(5 / 6)$ is the gamma function of $5 / 6$. $(\Gamma(5 / 6)=1.1288$.) All of the other terms in the equation have been previously defined. Equation 3 is the minimum value of beam heating possible for a smooth crack free bore tube surface. An expression for the transition from ordinary skin effect heat to anomalous skin effect heat can be found in references [6] and [7].

When one looks at the heating per unit length equations one finds that beam heating is proportional to the machine circumference divided by the number of bunches and the beam current I squared. The beam heating is inversely proportional to the beam gap $\mathrm{G}$. The beam heating is also inversely proportional to the beam bunch length to either the $3 / 2$ power or the $5 / 3$ power depending on which skin effect dominates.

\section{CAN ONE USE SMALL COOLERS TO COOL INSERTION DEVICES?}

Small gap insertion devices will have a relatively low mass. The physical cross-section dimensions of the insertion device are proportional to the magnet gap. As result, the mass of the device is well below the upper limit of the cold mass that can be cooled using small $4 \mathrm{~K}$ coolers. As a rule of thumb, the upper limit for the $4 \mathrm{~K}$ heat load is about $5 \mathrm{~W}$ (using multiple coolers). The upper limit of the $60 \mathrm{~K}$ heat load into the coolers is dependent on the total current entering the device from room temperature. The leads from $60 \mathrm{~K}$ to $4 \mathrm{~K}$ can be high temperature superconductor (HTS) leads and the heat leak for these leads can be quite low (about $0.4 \mathrm{~W}$ per $1000 \mathrm{~A}$ lead pair). The leads from $300 \mathrm{~K}$ to $60 \mathrm{~K}$ are copper leads that conduct heat into the $60 \mathrm{~K}$ stage of a two-stage cooler. The conduction heat load into the first stage of a cooler is about $70 \mathrm{~W}$ per 1000 A lead pair. Liquid nitrogen can be used to intercept heat coming down the room temperature leads. In general, small coolers can be used to cool the insertion device if the heat load at $4 \mathrm{~K}$ is less than $5 \mathrm{~W}$ and the current entering the device is less than $1800 \mathrm{~A}$. If liquid nitrogen can be used to intercept heat coming down the lead, the upper current limit can be extended to $4000 \mathrm{~A}$. An insertion device that operates in persistent mode, can operate at currents a factor of two higher (without retracting the leads) than a device that is powered continuously from an external power supply. If one is allowed to operate an insertion magnet in persistent mode and one is allowed to retract the leads, the upper current limit for cooling the device using small $4 \mathrm{~K}$ coolers is very high.

For modern light sources with undulators, $\mathrm{G}$ can be small while the beam current is high and the beam bunch length is very short, so it is easy to see how the beam heating term can be quite large (up to $50 \mathrm{~W}$ ). If the beam tube is at $4.4 \mathrm{~K}$, the use of small cryogenic coolers will be precluded unless the beam current is low so that beam heating is small. As a result, there may be a trade off concerning the cost of refrigeration and increasing the current density in the superconducting coils generating the insertion device magnetic field. When beam currents are low and $\mathrm{G}$ is small (say $<5 \mathrm{~mm}$ ) one may be able to run the beam tube at $4.4 \mathrm{~K}$ in order to improve the efficiency of the superconducting coils in the insertion device. Whether or not small coolers can be used depends on the heat load into the beam tube by conduction and radiation. For devices with $\mathrm{G}>8$ to $10 \mathrm{~mm}$, operating the insertion with a beam tube at say $50 \mathrm{~K}$ may be desirable, because one or two small single-stage coolers can remove the heat in the beam tube while the magnet itself is cooled with two-stage $4 \mathrm{~K}$ coolers. 
LBNL has used several Sumitomo RDK-415D coolers to cool superconducting magnets. Tests of the cooler done at LBNL show general agreement with the standard diagram for that cooler [9]. It should be pointed out that Cryomech produces a $4.2 \mathrm{~K}$ pulse tube cooler. These machines do not yet produce as much refrigeration as a singe Sumitomo cooler, but this situation could change. Both the GM coolers and the pulse tube coolers are quite reliable. Since there is no moving mass in the cold head of a pulse tube cooler, the vibration of this type of machine is a couple of orders of magnitude lower. Both the GM coolers and the pulse tube coolers exhibit temperature oscillations in their second stage cold heads. The peak to peak amplitude of this temperature oscillation is of the order of 0.25 to $0.30 \mathrm{~K}$. Because the magnets in the insertion device cryostats have considerable mass (more than $100 \mathrm{~kg}$ ), the variation of the cold head temperature should not be a problem. If the magnets are in contact with liquid helium, cold head temperature oscillation will never be a problem as long as the $\Delta \mathrm{T}$ between the magnet and the cold head is low.

\section{CONNECTION OF THE COOLER COLD HEAD TO THE COLD MASS}

One of the most import things that one can do when one cools an insertion device is to properly connect the cold head of the cooler (or coolers) to the device. First, the connection between the cold head and the device must not transfer vibration from the cold head to the device. Second, the temperature drop between the insertion device and the cold head must be minimized, in order for the performance of the magnet not to be compromised.

In general, there are two ways to connect the cold head to the load. One may run the load completely dry with no liquid helium in the magnet. One may immerse the device in liquid helium, but one must connect the cold head to the liquid helium in such a way that the $\Delta \mathrm{T}$ between the coil high field zone and the cold head is minimized. Here the authors describe two methods for vibration isolation and connection of the cold head to the load. The two methods are; 1) connection of the cold head to the cold mass directly through a flexible copper strap, and 2) connection of the cold head to the cold mass immersed in liquid helium through a gravity separated heat pipe.

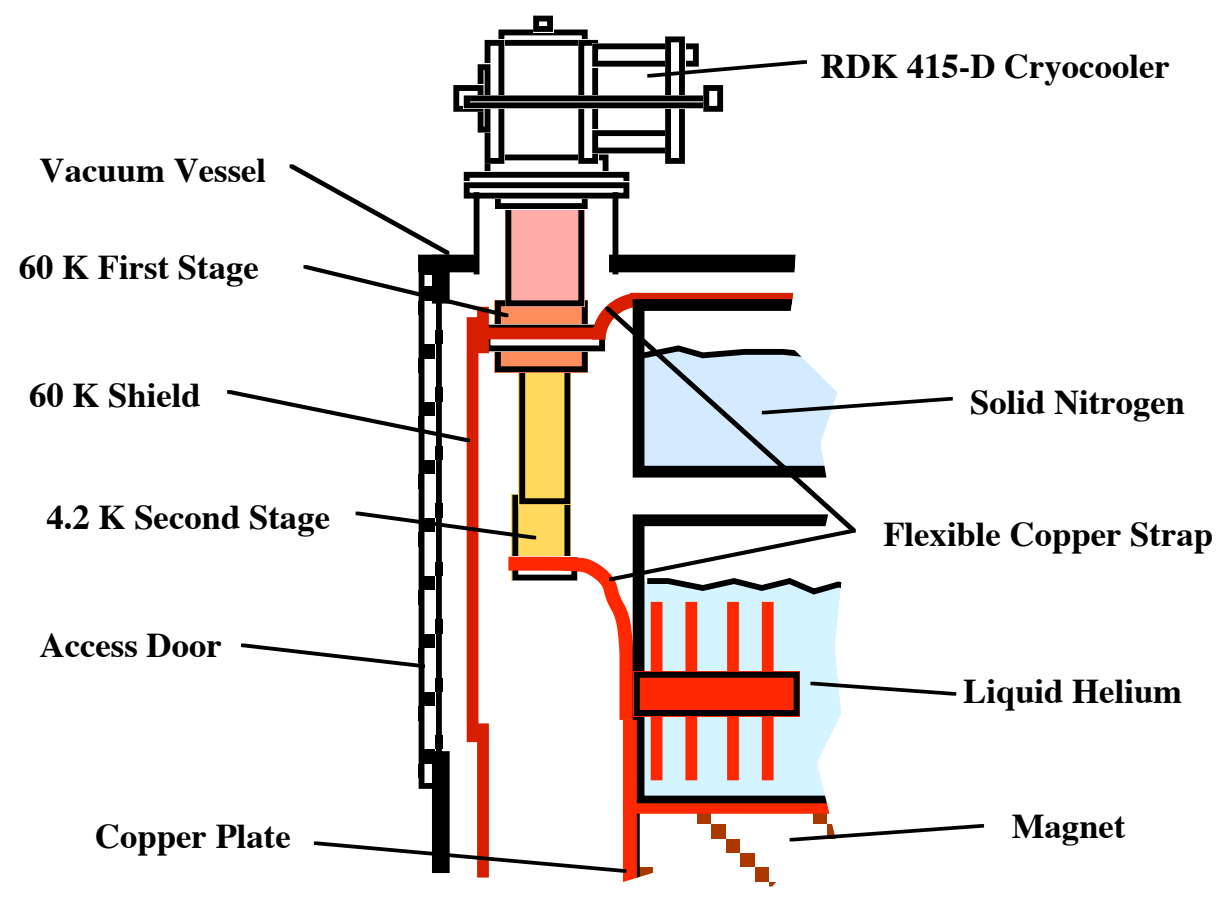

FIGURE 2. Connection of a cooler to an insertion device through a flexible copper strap. Note: Copper straps are used to connect both stages of the cooler to the load and the cryogen vessels. 
FIG 2 shows how a Sumitomo RKDK-415D cooler can be connected to an insertion magnet. The magnet cryostat has a tank of liquid helium and liquid nitrogen that acts as a buffer between the cooler cold head and the load to be cooled. If there is a power failure (or cooler failure), the cryogen reservoirs can keep the magnet cold until the problem can be dealt with.

In order for the copper strap to act as an effective vibration isolation system, the strap must be finely divided, so that it is flexible. The biggest problem with using a copper strap to carry the cold from the magnet to the cooler cold head is the $\Delta \mathrm{T}$ between the load being cooled and the cold head. In most cases, a $\Delta \mathrm{T}$ of 10 to $15 \mathrm{~K}$ between the heat load and the first stage cold head is entirely acceptable. The acceptable $\Delta \mathrm{T}$ between a magnet and the second stage cold head is nearly always less than $0.3 \mathrm{~K}$.

One can estimate the $\Delta \mathrm{T}_{\mathrm{Cu}}$ between the load and the cold head of the cooler by using the following expression;

$$
\Delta T_{C u}=\frac{Q}{A_{C u}} \frac{L_{C u}}{k_{C u}}
$$

where $\mathrm{Q}$ is the heat being transferred through the strap; $\mathrm{A}_{\mathrm{Cu}}$ is the cross-section area of the strap; $\mathrm{L}_{\mathrm{Cu}}$ is the length of the copper strap; and $\mathrm{k}_{\mathrm{Cu}}$ is the thermal conductivity of the strap. An approximate value of $\mathrm{k}_{\mathrm{Cu}}=\left(\mathrm{LT}_{\mathrm{Cu}}\right) / \rho_{\mathrm{Cu}}$, where $\mathrm{T}_{\mathrm{Cu}}$ is the strap average temperature; $\mathrm{L}$ is the Lorenz number $\left(\mathrm{L}=2.45 \times 10^{-8} \mathrm{~W} \Omega \mathrm{K}^{-2}\right)$; and $\rho_{\mathrm{Cu}}$ is the strap electrical resistivity. From Equation 4 , it is clear that to minimize the $\Delta \mathrm{T}$ one wants to increase the cross-section area of the strap, decrease the length of the strap, and maximize the strap thermal conductivity. In order to increase the strap thermal conductivity, one must use a copper with a large RRR and one must make sure that the strap is not cold worked so that the RRR stays high with time.

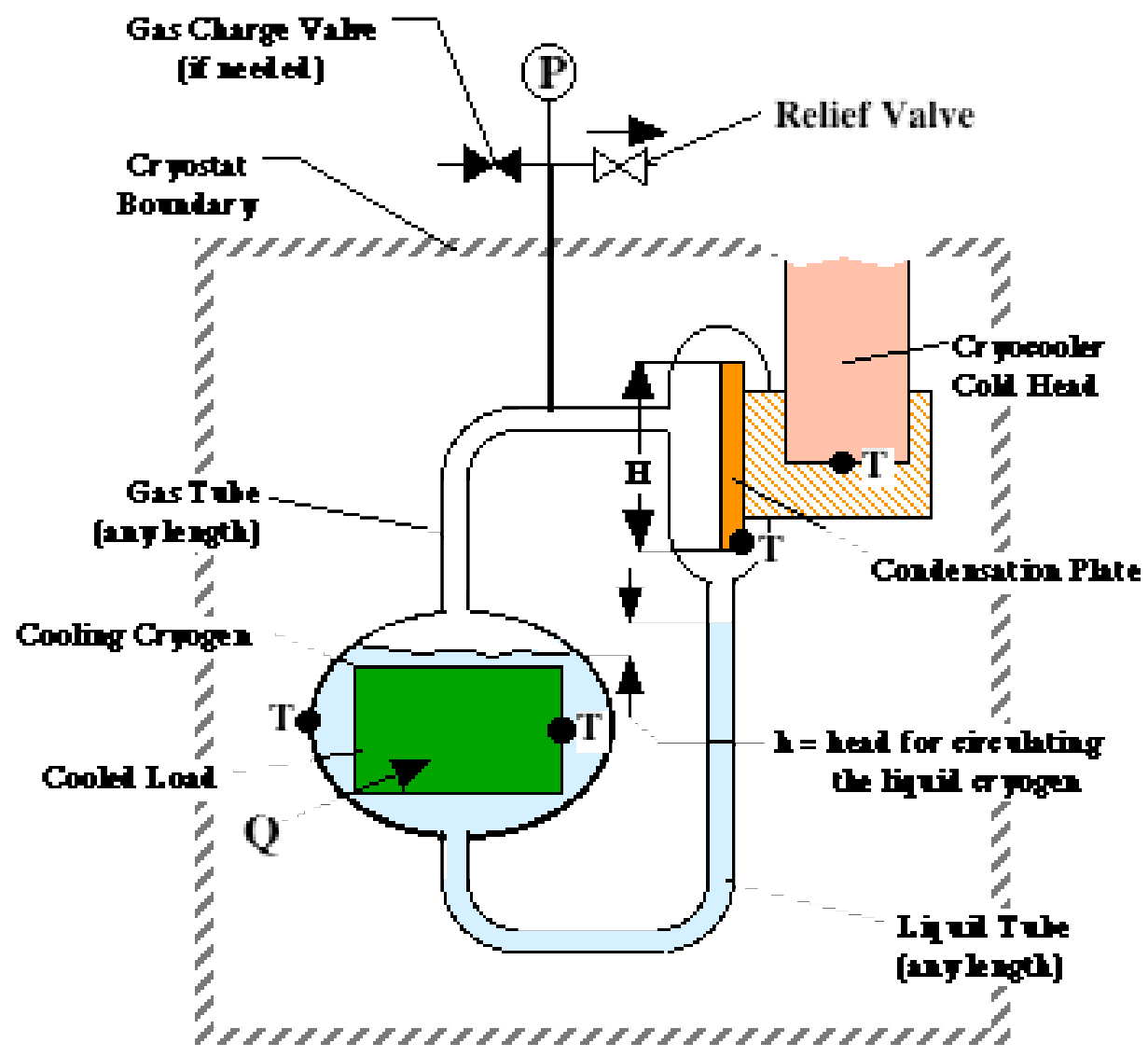

FIGURE 3. A schematic representation of a gravity loop heat pipe system to transfer heat from the load to the cooler cold head. (Note: the heat pipe system shown is an open cycle system that allows helium to be added to the system. Heat pipes that are completely sealed can also be built.) 
FIG 3 shows a schematic representation of a gravity separated heat pipe that can be used to transfer heat from the insertion device (cooled load) to liquid cryogen (helium) to the cold head of a cooler (in this case the second stage cold head). A small amount of helium is boiled on the cooled load and the walls of the liquid cryostat. The gas is carried to a condensation plate through a flexible gas tube. The gas is condensed on the condensation plate and the liquid flows back to the liquid bath through the liquid tube. This type of device for transferring heat from a load to the cold head has been used on the Venus magnet at LBNL with a total $\Delta \mathrm{T}$ from the cold head to the magnet of less than $0.05 \mathrm{~K}[10]$.

The $\Delta \mathrm{T}$ from the load to the cold head is the sum of the nucleate boiling temperature drop $\Delta \mathrm{T}_{\mathrm{b}}$ and the condensation temperature drop $\Delta \mathrm{T}_{\mathrm{C}}$. The condensation temperature drop can be calculated with the following expression [11];

$$
\Delta T_{c}=\left[\left(\frac{Q}{A}\right)_{c}\left(\frac{\mu_{l} H}{\rho_{l}^{2} g h_{f g} k_{l}^{3}}\right)^{0.25}\right]^{\frac{4}{3}},
$$

where $(\mathrm{Q} / \mathrm{A})_{\mathrm{c}}$ is the heat transferred per unit area across the condenser; $\mu_{1}$ is the viscosity of the liquid; $H$ is the height of the condensing plate; $\rho_{1}$ is the density of the liquid; $g$ is the acceleration of gravity; $h_{f g}$ is the heat of vaporization of the fluid; and $k_{1}$ is the liquid thermal conductivity. For condensing liquid helium, one must have a value of $(\mathrm{Q} / \mathrm{A})_{\mathrm{c}}<40 \mathrm{~W} \mathrm{~m}^{-2}$ on the condenser surface. Extending the condenser surface is nearly always a good idea.

The boiling surface $\Delta \mathrm{T}$ can be estimated by using methods outlined by Frederking in his 1962 paper [12]. An expression for the temperature drop $\Delta \mathrm{T}_{\mathrm{b}}$ from a device to liquid helium through nucleate boiling at $4.2 \mathrm{~K}$ can be given by the following expression [6], [13];

$$
\Delta T_{b}=\left[\frac{(Q / A)_{b}}{(Q / A)_{n p}}\right]^{0.4} \Delta T_{n p}
$$

where $(\mathrm{Q} / \mathrm{A})_{\mathrm{b}}$ is the heat transfer per unit area on the boiling surface (the magnet surface), $(\mathrm{Q} / \mathrm{A})_{\mathrm{np}}$ is the peak nucleate boiling flux for the fluid from a surface facing upward (For helium at $4.2 \mathrm{~K}(\mathrm{Q} / \mathrm{A})_{\mathrm{np}}=8000 \mathrm{~W} \mathrm{~m}^{-2}$.); and $\Delta \mathrm{T}_{\mathrm{np}}$ is the peak temperature drop associated with peak nucleate boiling on a open surface facing upward (For helium $\Delta \mathrm{T}_{\mathrm{np}}=0.8 \mathrm{~K}$.). In

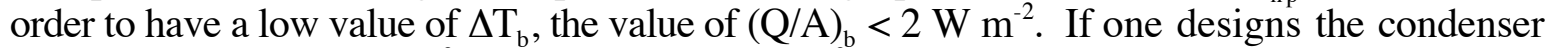
so that $(\mathrm{Q} / \mathrm{A})_{\mathrm{c}}=40 \mathrm{~W} \mathrm{~m} \mathrm{~m}^{-2}$ and $(\mathrm{Q} / \mathrm{A})_{\mathrm{b}}=2 \mathrm{~W} \mathrm{~m}^{-2}$ for the magnet the total temperature drop from the magnet to the cold head is less than $0.05 \mathrm{~K}$.

It is useful to note that equations 5 and 6 do not have any dependence on the distance between the magnet and the condenser surface attached to the cooler cold head. The pipes connecting the magnet cryostat to the condensation chamber must be large enough to carry the gas and liquid flow with a reasonably small pressure drop. (The pipe flow $M_{f}=Q / h_{f g}$.). As long as the pipes are sized correctly, the cooler cold head can be separated from the magnet by a meter or more.

For cooling insertion device magnets, the heat pipe offers the following advantages over direct connection of the cold head to the magnet or connection to the magnet through a copper strap; 1) Excellent vibration isolation can be obtained because the pipes can be made flexible. 2) The temperature oscillation in the cold head can be completely filtered out. The magnet will operate at a constant temperature that is dependent on the total heat load going into the cooler (coolers). Heat pipes permit multiple coolers to be connected to the load. 3) When a cooler is shut off the heat pipe behaves like thermal diode. If the cold head is warmer than the magnet, the heat transfer from the cold head to the magnet will be poor. One can design the system to have very poor heat transfer from the cold head to the helium bath. This allows a system to be operated with several coolers so that one cooler can be shut down for maintenance of a compressor that is some distance from the cold head. 


\section{CONCLUDING COMMENTS}

Cooling of superconducting insertion devices is an important issue. Cooling may become a dominant issue for an undulator, because the undulator has a small period and a small magnet gap. The small magnet gap may require that the beam tube in the undulator be at cryogenic temperatures. The temperature of the undulator beam pipe becomes a contributing issue to the overall cooling of the device.

Undulators have small gaps; they are often on light sources with large beam currents and short beam bunch lengths. All of these things will cause heating in the beam tube. At low beam tube temperatures, the anomalous skin effect becomes dominant. The surface condition of the inner wall of the beam tube will affect the heating due to the storage ring beam.

Most room temperature bore insertion magnets can be cooled using small cryogenic coolers. When the beam tube is in the temperature range from 30 to $70 \mathrm{~K}$, an extra single stage cooler may be needed in order to cool the beam tube. Undulators with a $4 \mathrm{~K}$ bore will have to be cooled using conventional helium refrigerators unless there is very little beam heating due to the image currents in the beam pipe.

The connection of the insertion device magnet to the cooler is very important. One must have a low $\Delta \mathrm{T}$ (say less than $0.3 \mathrm{~K}$ ) between the cold head and the load being cooled. Heat pipes are a good method for getting a low $\Delta \mathrm{T}$ between the magnet and the cold head of a small cooler. The use of a heat pipe permits one to move the cold head of the cooler some distance from the device being cooled.

\section{ACKNOWLEDGEMENTS}

This work was performed at the Lawrence Berkeley National Laboratory with the support of the Office of Industrial Technologies, United States Department of Energy under DOE contract DE-AC03-76SF00098.

\section{REFERENCES}

1. M. A. Green, E. H. Hoyer, et al., "Refrigeration Options for the Advanced Light Source Superbend Dipole Magnets," Advances in Cryogenic Engineering 45, p1363, Plenum Press, New York (1999)

2. "Review of Particle Properties," Review of Modern Physics 48, No. 2, Part II, April 1976

3. John D Jackson, Classical Electrodynamics, $3^{\text {rd }}$ Edition, John Whiley and Sons Inc. New York

4. Center for X-ray Optics, Advance Light Source, "X-ray Data Booklet," $2^{\text {nd }}$ Edition, LBNL, Jan. 2001

5. D. R. Dietderich, R. M. Scanlan, S. A. Gourlay et. al. Private communication concerning the cold test of a short section of a $\mathrm{Nb}_{3} \mathrm{Sn}$ undulator.

6. M. A. Green, "Cryogenic Refrigeration Requirements for Superconducting Insertion Devices in a Light Source," Lawrence Berkeley National Laboratory Report LBNL-52578, (August 2003)

7. W. Chou and F. Ruggiero, "Anomalous Skin Effect and Resistive Wall Heating," CERN-LHC Note 2, September 8, 1995

8. A. B. Pippard, Proceedings of the Royal Society (London) A191, p 385 (1947)

9. J. Zbasnik, M. A. Green, et al., "Tests of a GM Cryo-cooler and High Tc Leads for the ALS Superbend Magnets," Advances in Cryogenic Engineering 45, p 635, Plenum Press, New York, (1999)

10. C. E. Taylor, S. R. Abbott, D Leitner, et al., "An Efficient Cooling Loop for Connecting a Cryocooler to a Helium Reservoir," Advances in Cryogenic Engineering 49 (this volume), AIP Press, (2003)

11. Frank Kreith, Principles of Heat Transfer, International Textbook Co., Scranton PA. (1958)

12. T. K. H. Frederking, "Peak Heat Flux and Temperature Difference in Nucleate Boiling of Liquefied Gasses," Advances in Cryogenic Engineering 8. P 489, Plenum Press, New York, (1962)

13. M. A. Green, "Integration of Liquid Cryogen Cooling and Cryo-coolers with Superconducting Electronic Systems," Superconducting Science and Technology, ISEC-2003 Special Issue, (2003) 


\section{DISCLAIMER}

This document was prepared as an account of work sponsored by the United States Government.

While this document is believed to contain correct information, neither the United States Government nor any agency thereof, nor The Regents of the University of California, nor any of their employees, makes any warranty, express or implied, or assumes any legal responsibility for the accuracy, completeness, or usefulness of any information, apparatus, product, or process disclosed, or represents that its use would not infringe privately owned rights. Reference herein to any specific commercial product, process, or service by its trade name, trademark, manufacturer, or otherwise, does not necessarily constitute or imply its endorsement, recommendation, or favoring by the United States Government or any agency thereof, or The Regents of the University of California. The views and opinions of authors expressed herein do not necessarily state or reflect those of the United States Government or any agency thereof, or The Regents of the University of California. 